\title{
Calcineurin Controls Expression of EAAT1/GLAST in Mouse and Human Cultured Astrocytes through Dynamic Regulation of Protein Synthesis and Degradation
}

\author{
Giulia Dematteis ${ }^{1}$, Elena Restelli ${ }^{2}$, Roberto Chiesa ${ }^{2}{ }^{\circledR}$, Eleonora Aronica ${ }^{3,4}$, \\ Armando A Genazzani ${ }^{1}$, Dmitry Lim ${ }^{1, *(D)}$ and Laura Tapella ${ }^{1}$ (D) \\ 1 Department of Pharmaceutical Sciences, Università del Piemonte Orientale "Amedeo Avogadro", \\ 28100 Novara, Italy; giu.dematteis@libero.it (G.D.); armando.genazzani@uniupo.it (A.A.G.); \\ laura.tapella@uniupo.it (L.T.) \\ 2 Department of Neuroscience, Istituto di Ricerche Farmacologiche Mario Negri IRCCS, 20156 Milano, Italy; \\ elena.restelli@marionegri.it (E.R.); roberto.chiesa@marionegri.it (R.C.) \\ 3 Amsterdam UMC, University of Amsterdam, Department of (Neuro) Pathology, \\ 1105 AZ Amsterdam, The Netherlands; e.aronica@amsterdamumc.nl \\ 4 Stichting Epilepsie Instellingen Nederland (SEIN), 2103 Heemstede, The Netherlands \\ * Correspondence: dmitry.lim@uniupo.it; Tel.: +39-0321-375822
}

Received: 12 March 2020; Accepted: 21 March 2020; Published: 23 March 2020

\begin{abstract}
Alterations in the expression of glutamate/aspartate transporter (GLAST) have been associated with several neuropathological conditions including Alzheimer's disease and epilepsy. However, the mechanisms by which GLAST expression is altered are poorly understood. Here we used a combination of pharmacological and genetic approaches coupled with quantitative PCR and Western blot to investigate the mechanism of the regulation of GLAST expression by a $\mathrm{Ca}^{2+} /$ calmodulin-activated phosphatase calcineurin $(\mathrm{CaN})$. We show that treatment of cultured hippocampal mouse and fetal human astrocytes with a CaN inhibitor FK506 resulted in a dynamic modulation of GLAST protein expression, being downregulated after $24-48 \mathrm{~h}$, but upregulated after 7 days of continuous FK506 (200 nM) treatment. Protein synthesis, as assessed by puromycin incorporation in neo-synthesized polypeptides, was inhibited already after $1 \mathrm{~h}$ of FK506 treatment, while the use of a proteasome inhibitor MG132 $(1 \mu \mathrm{M})$ shows that GLAST protein degradation was only suppressed after 7 days of FK506 treatment. In astrocytes with constitutive genetic ablation of $\mathrm{CaN}$ both protein synthesis and degradation were significantly inhibited. Taken together, our data suggest that, in cultured astrocytes, CaN controls GLAST expression at a posttranscriptional level through regulation of GLAST protein synthesis and degradation.
\end{abstract}

Keywords: astrocytes; calcineurin; GLAST; protein synthesis; protein degradation; proteostasis

\section{Introduction}

Glutamate is a principal excitatory neurotransmitter in the central nervous system (CNS) and its correct homeostasis, which includes uptake and release, is of paramount importance for correct function of the CNS. Released by glutamatergic terminals at the excitatory synapses, glutamate acts on both ionotropic and metabotropic receptors to transmit signals and induce plasticity [1]. Uptake of glutamate is mediated by a family of five excitatory amino acid transporters (EAAT1-5) [1] differentially expressed in different brain regions and cell types, all acting with the same stoichiometry: Importing one glutamate molecule by the co-transport of three sodium ions and one proton, while exporting 
one potassium ion [2]. Astrocytes, being the principal glutamate-metabolizing cells in the CNS, are endowed by two high affinity glutamate transporters, glutamate/aspartate transporter EAAT1/GLAST and glial high affinity glutamate transporter 1 EAAT2/Glt-1, which are preferentially expressed at the membrane surface [3]. In healthy adult hippocampus EAAT2/Glt-1 is preferentially expressed, while in cultured hippocampal astrocytes EAAT1/GLAST becomes predominant [4-6].

Alterations of Glt-1 and GLAST expression and activity have been associated with many neurological disorders such as schizophrenia, epilepsy and Alzheimer's disease (AD) [7,8]. Therefore, understanding of how the EAATs expression is regulated is important for identification of therapeutic targets to treat pathologies correlated with alterations of glutamate transporters.

Expression of Glt-1 and GLAST has been shown to be regulated at both transcriptional and translational levels depending on experimental and/or pathological context $[9,10]$. At the transcriptional level Gtl-1 has been shown to be inversely regulated by calcineurin ( $\mathrm{CaN})$-nuclear factor of activated T-cells (NFAT) axis [11,12]. Whether GLAST is also regulated by $\mathrm{Ca}^{2+}$ and $\mathrm{CaN}$ is currently not known.

$\mathrm{CaN}, \mathrm{Ca}^{2+} /$ calmodulin-activated phosphatase, is a heterodimer, composed of a catalytic subunit CaNA and an obligatory regulatory subunit CaNB. Cooperative binding of $\mathrm{Ca}^{2+}$ to $\mathrm{CaNB}$ and of $\mathrm{Ca}^{2+} /$ calmodulin complex to CaNA leads to displacement of the auto-inhibitory domain permitting the interaction of $\mathrm{CaN}$ with its substrates. CaN activates gene transcription through direct activation of NFAT [13] and modulation of the activation cascade of NF-kB [14]. CaN is also able to regulate activity, interaction, or location of target proteins directly through dephosphorylation [15].

Recently we have reported that in cultured hippocampal astrocytes from an astroglial CaN knock-out mouse, GLAST protein was upregulated while mRNA levels were not changed [6]. Thus, we thought to investigate the mechanism of CaN-dependent up-regulation of GLAST in cultured astrocytes. Our results suggest that $\mathrm{CaN}$ in astrocytes regulates expression of GLAST at the posttranscriptional level through dynamic regulation of protein synthesis and degradation.

\section{Results}

\subsection{Pharmacological CaN Inhibition Results in a Dynamic Modulation of GLAST Protein in Both Mouse and Human Astrocytes}

Starting from the observation of increased GLAST expression in cultured hippocampal astrocytes with a genetic deletion of $\mathrm{CaN}$ (CaN-KO) [6] we investigated if pharmacological inhibition of $\mathrm{CaN}$ with the specific and clinically relevant inhibitor FK506 (200 nM) would result in GLAST overexpression. Surprisingly, after 1 or 2 days of treatment GLAST was, instead, downregulated (Figure 1A). We have reasoned that in CaN-KO astrocytes, the upregulation of GLAST would represent an end-point of the effect of CaN deletion. Therefore, we followed GLAST expression until 7 days of FK506 treatment and found that at 7 day GLAST was significantly up-regulated resembling the upregulation observed in $\mathrm{CaN}-\mathrm{KO}$ astrocytes (Figure 1A). In an immunocytochemical analysis the downregulation of GLAST at 1 or 2 days of FK506 treatment was not evident (not shown) likely due to the low basal level of GLAST expression, while at the 7th day of treatment GLAST fluorescence was strongly increased (Figure 1B). Next we investigated if the biphasic dynamics of GLAST expression upon CaN inhibition was due to changes in mRNA levels, e.g., if CaN regulated GLAST expression at transcriptional level. Quantitative PCR results show that the mRNA levels of GLAST were not different at any time point of FK506 treatment (Figure 1C), corroborating the absence of transcriptional regulation in CaN-KO astrocytes [6].

To consolidate this finding and to demonstrate that the effect of $\mathrm{CaN}$ inhibition was not limited exclusively to mouse astrocytes, we treated with FK506 (200 nM) fetal human cultured astrocytes. The observed dynamics of GLAST expression during 7 days treatment was very similar to that of mouse hippocampal astrocytes showing significant downregulation at 1 st and 2 nd days and upregulation after 7 days of treatment (Figure 2A). Similarly to mouse astrocytes, immunofluorescent analysis revealed strong increase in immunoreactivity to GLAST at 7 days (Figure 2B), while mRNA levels did not change at any time-point (Figure 2C). 

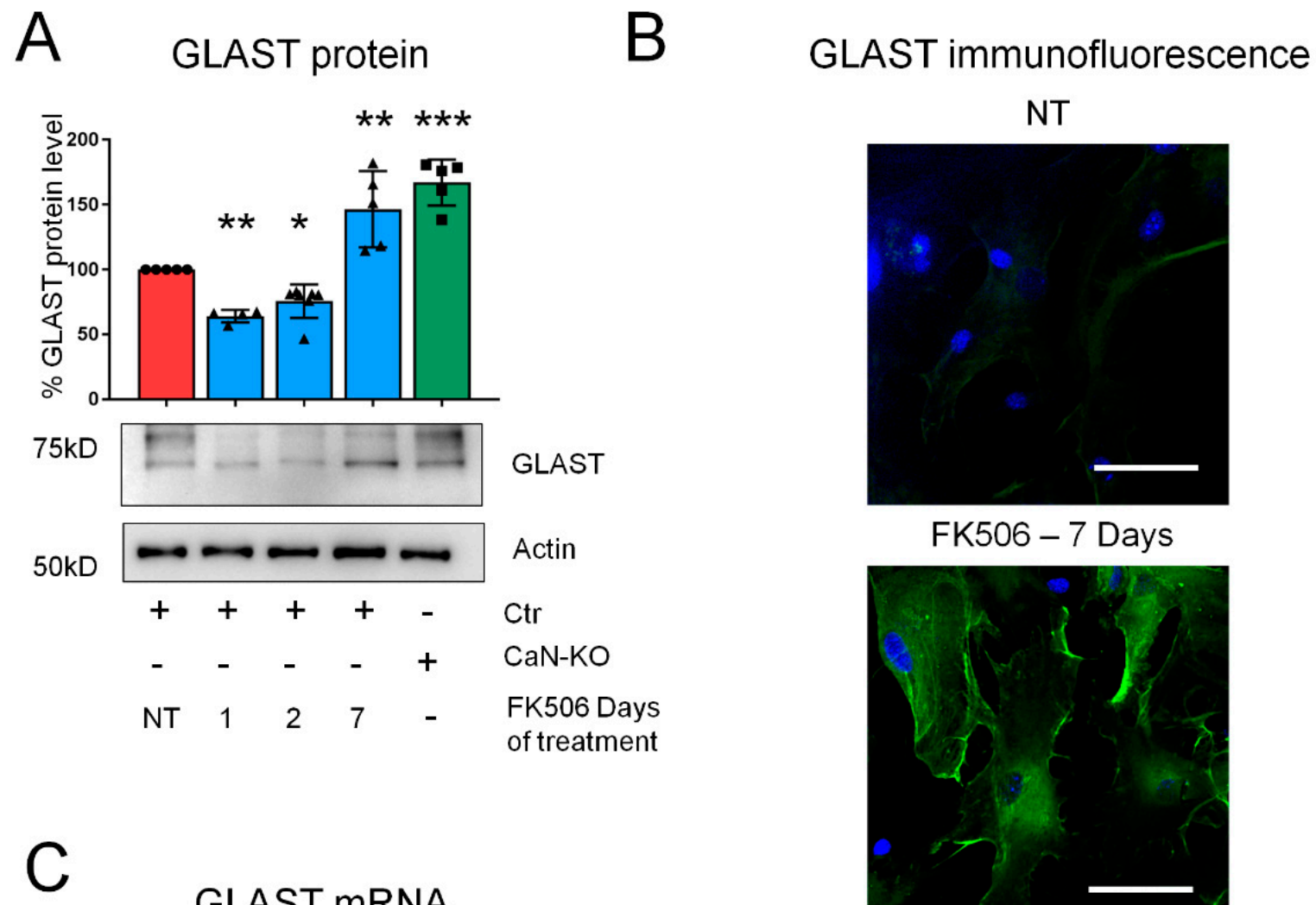

\section{GLAST mRNA}

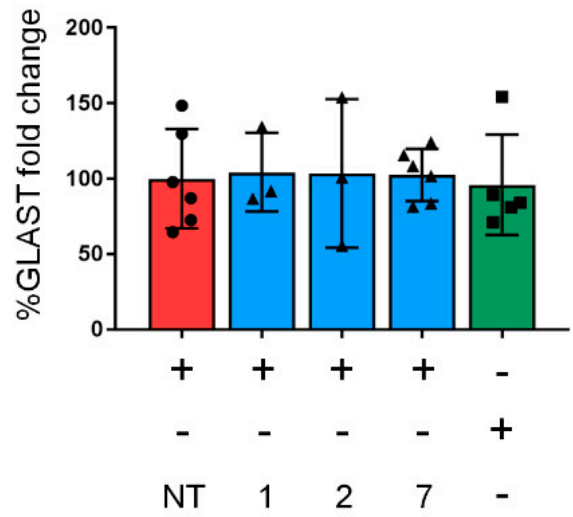

NT

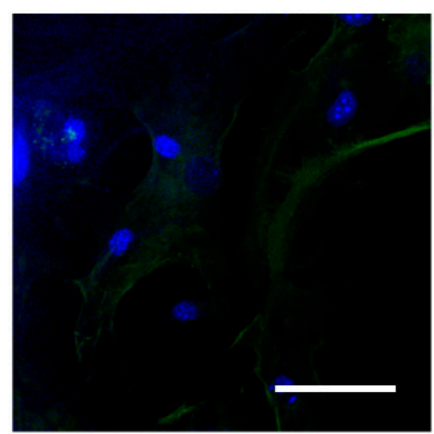

FK506 - 7 Days

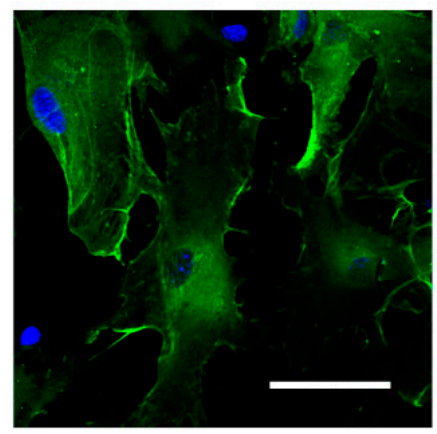

CaN-KO

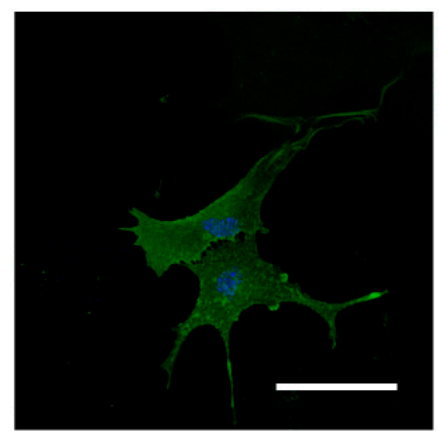

Figure 1. CaN inhibition dynamically regulates GLAST expression in mouse hippocampal astrocytes. (A) Glutamate/aspartate transporter (GLAST) protein expression in primary cultures of hippocampal astrocytes from control not-treated (Ctr, NT) Ctr astrocytes treated with FK506 200nM for 1, 2 and 7 days and of cultured astrocytes from mice with an astrocyte-specific deletion of $\mathrm{Ca}^{2+} /$ calmodulin-activated phosphatase calcineurin ( $\mathrm{CaN}-\mathrm{KO})$. Data are expressed as mean $\pm \mathrm{SD}, 5$ independent cultures were used for each condition. ${ }^{*} p<0.05 ;{ }^{* *} p<0.01$; ${ }^{* * *} p<0.001$, one way ANOVA with Tukey post hoc test. (B) Immunofluorescence anti-GLAST images of Ctr-NT, Ctr treated with FK506 $200 \mathrm{nM}$ for 7 days and CaN-KO. Green, GLAST; blue, DAPI (4',6-diamidino-2-phenylindole). Bar, $50 \mu \mathrm{m}$. (C) Real-time PCR of GLAST from primary astrocytes from Ctr-NT, Ctr treated with FK506 200 nM for 1, 2, and 7 days, and from astrocyte-specific CaN-KO mice. Values represent mean $\pm \mathrm{SD} \Delta \mathrm{C}(\mathrm{t})$ of gene/S18 of 6 independent cultures for each condition. 

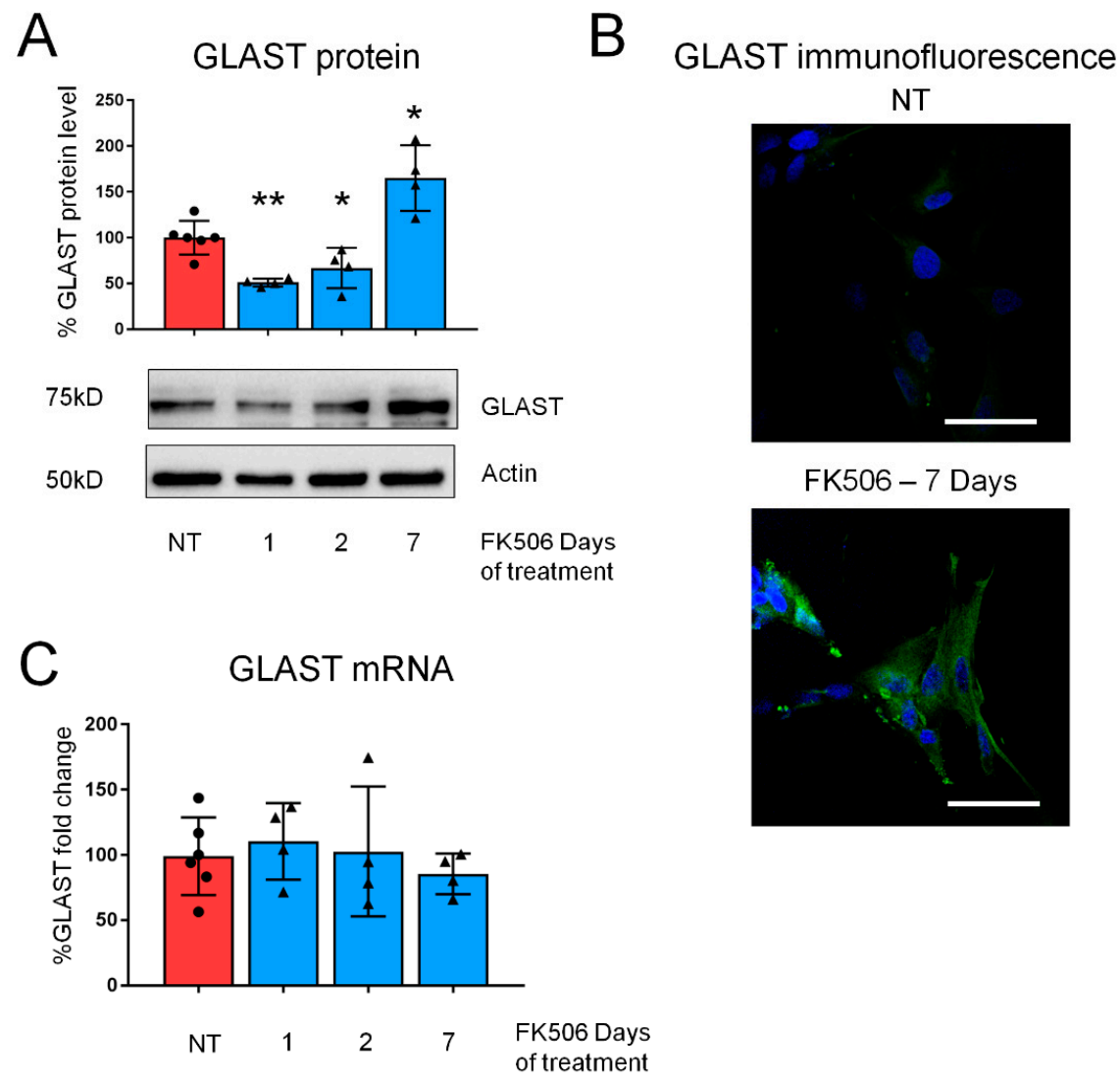

Figure 2. CaN inhibition dynamically regulates GLAST expression human astrocytes. (A) GLAST protein expression in primary cultures of human astrocytes untreated and treated with FK506 (200 nM) for 1,2 , and 7 days. Data are expressed as mean \pm SD. ${ }^{*} p<0.05 ;{ }^{* *} p<0.01$; one way ANOVA with Tukey post hoc test. (B) Immunofluorescence images of human primary astrocytes, untreated and treated with FK506 for 7 days, stained with anti-GLAST antibody (green). Nuclei are stained with DAPI (blue). Bar, $50 \mu \mathrm{m}$. (C) Real-time PCR of GLAST from human primary astrocytes, untreated and treated with FK506 (200 nM) for 1, 2 and 7 days. Values represent mean \pm SD $\Delta C(t)$ of gene/S18 of 4 independent experiments for each condition.

2.2. CaN Modulates GLAST Protein Expression through the Regulation of the Equilibrium between Protein Synthesis and Degradation

Given the absence of alterations in GLAST mRNA levels we reasoned that the dynamic modulation of GLAST protein may be a result of alterations of protein synthesis rate. To investigate this, we employed a recently developed method of surface sensing of translation (SUnSET) which is based on puromycin incorporation in neo-synthetized peptides [16] with consequent detection by anti-puromycin immunoreactivity either by immunoblot or immunofluorescence. We found that incorporation of puromycin was drastically inhibited already $1 \mathrm{~h}$ after addition of FK506 (Figure 3A). Longer FK506 incubation somewhat resulted in the appearance of a smear in anti-puromycin probed membranes which rendered quantification of the band intensity unreliable. Nevertheless, we found that incorporation of puromycin in $\mathrm{CaN}-\mathrm{KO}$ astrocytes was also significantly inhibited (Figure 3A), suggesting that the impairment of protein synthesis in FK506-treated astrocytes was a result of inhibition of $\mathrm{CaN}$ activity. To follow the long-term effect of pharmacological CaN inhibition, we took advantage of immunofluorescent puromycin labelling. Interestingly, beginning from the second day of FK506 treatment, a drastic reduction of puromycin immunoreactivity was observed in all time points, including 7 days-time-point, suggesting an irreversible inhibition of proteins synthesis upon blockade of CaN activity (Figure 3B). In line with this, fluorescence intensity of anti-puromycin staining in CaN-KO astrocytes was also significantly lower compare to control/not-treated astrocytes. Thus, 
the downregulation of GLAST protein during the first days of FK506 treatment may be a result of the inhibited protein synthesis downstream of pharmacological inactivation of $\mathrm{CaN}$ in astrocytes. However, the upregulation of GLAST after long-term FK506 treatment or in astrocytes with KO of $\mathrm{CaN}$ was at odds with the inhibition of protein synthesis.
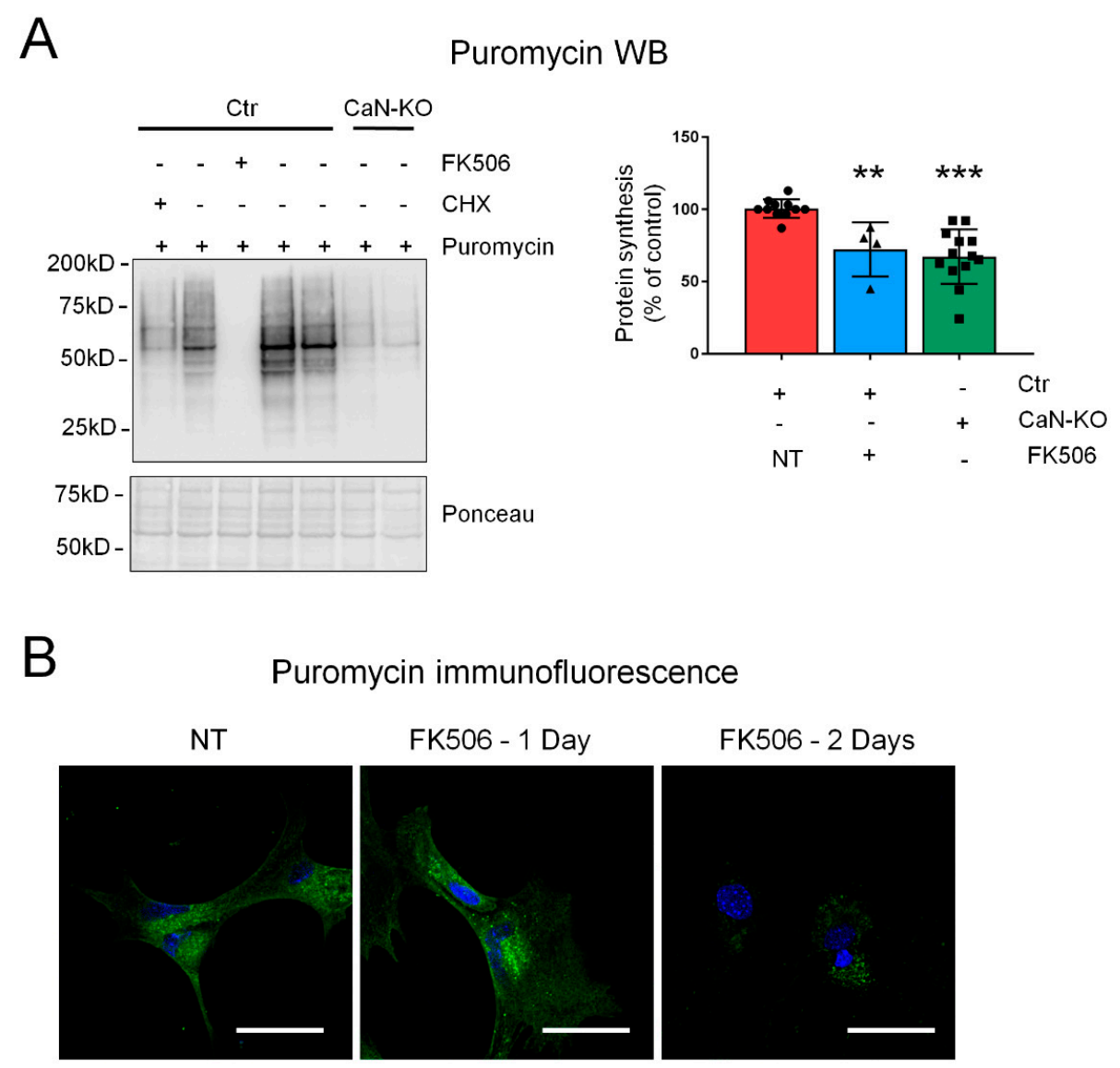

Puromycin immunofluorescence
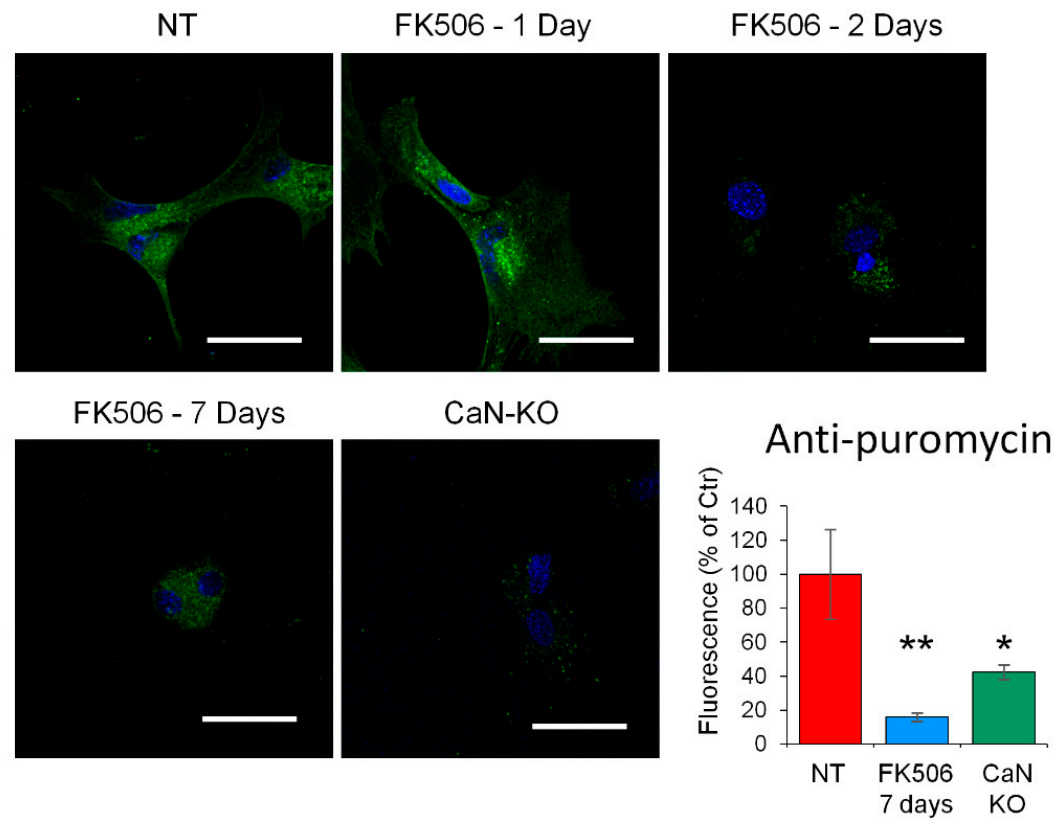

Figure 3. CaN inhibition permanently suppresses protein synthesis in cultured astrocytes. (A) Ctr and $\mathrm{CaN}-\mathrm{KO}$ primary astrocytes were pulsed with puromycin for $3 \mathrm{~h}$. Ctr were also treated with FK506 for $1 \mathrm{~h}$ to inhibit $\mathrm{CaN}$ activity. Where indicated, cycloheximide $(\mathrm{CHX}, 10 \mu \mathrm{M})$ was added ten minutes before adding puromycin. Anti-puromycin antibody was used to detect neo-synthesized peptides. Ponceau staining was used for band normalization. Histogram shows quantification of anti-puromycin detected bands. Data are expressed as mean \pm SD, 5, 4, and 11 independent cultures were used for Ctr, FK506 treated and CaN-KO samples, respectively. ${ }^{* *} p<0.01 ;{ }^{* *} p<0.001$, one way ANOVA with Tukey post hoc test. (B) Immunofluorescence images of Ctr, Ctr treated with FK506 (200 nM) for 1, 2 and 7 days and $\mathrm{CaN}-\mathrm{KO}$, stained with anti-puromycin antibody (green). Nuclei are stained with DAPI (blue). Bar, $25 \mu \mathrm{m}$. Data are expressed as mean $\pm \mathrm{SD}$; ${ }^{*} p<0.05 ;{ }^{* *} p<0.01$, one way ANOVA with Tukey post hoc test. 
Because of the amount of a protein in the cell, at a given transcriptional rate, is determined by the equilibrium of protein synthesis and protein degradation [17], we hypothesized that the augmented GLAST protein in concomitance with the inhibition of protein synthesis may result from the impairment of protein degradation system, namely proteasome [18]. To test this hypothesis, we treated astroglial primary cultures with MG132 $(1 \mu \mathrm{M}, 3 \mathrm{~h})$, a potent proteasome inhibitor [19]. Expectedly, treatment of control astrocytes with MG132 induced a significant increase in GLAST protein expression (Figure 4) as it did also after 1 day of FK506 treatment, indicating that proteasome was active in these conditions. However, neither in astrocytes at 7 th day of FK506 treatment nor in CaN-KO astrocytes, increase of GLAST expression was observed (Figure 4), suggesting that both long-term FK506 treatment and genetic $\mathrm{CaN}$ ablation resulted in inhibition of proteasome activity.

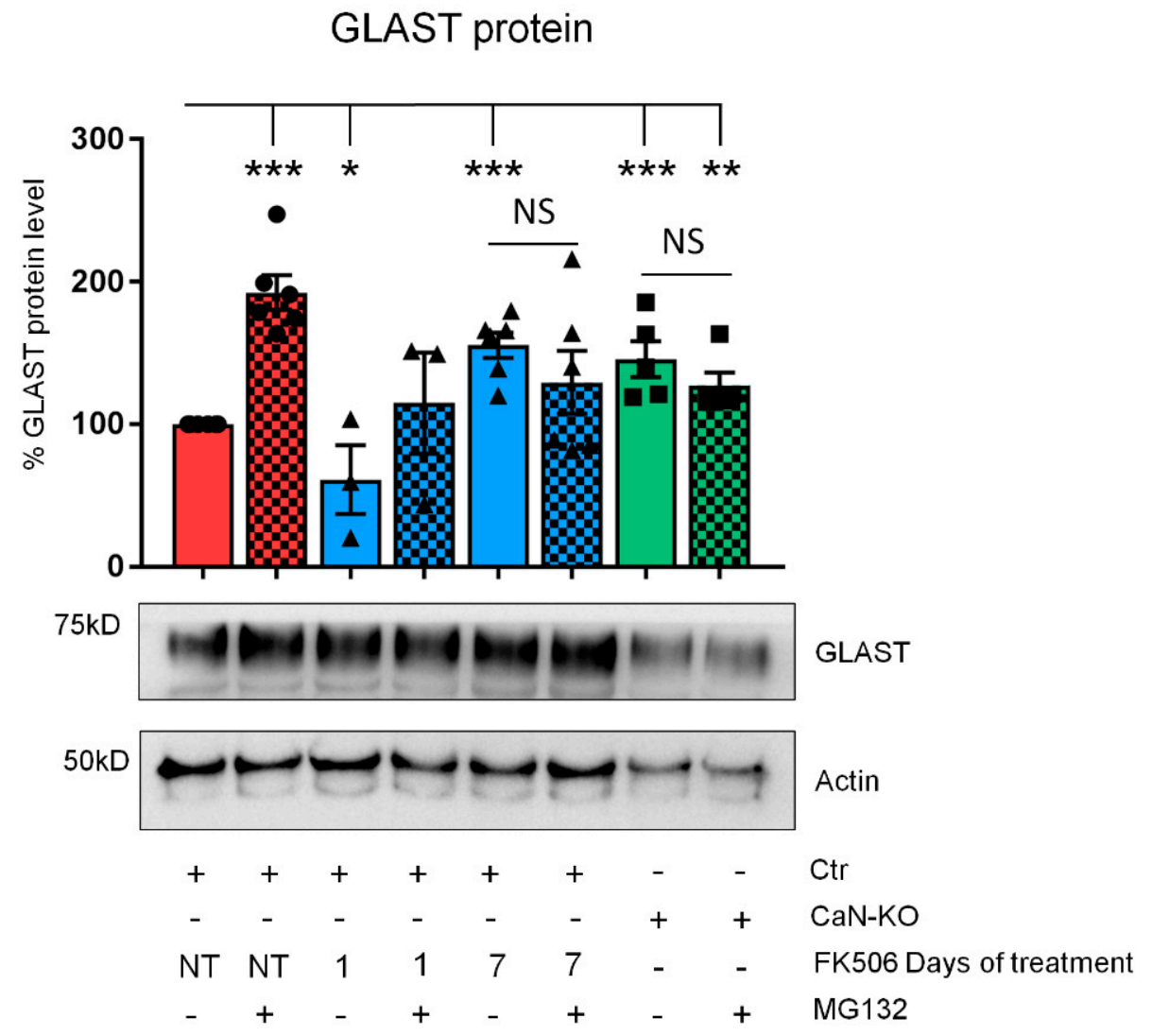

Figure 4. Long-term, but not short-term CaN inhibition suppresses GLAST protein degradation. GLAST protein expression in primary cultures of hippocampal astrocytes from Ctr, Ctr treated with FK506 (200 nM) for 1 and 7 days and from astrocytes-specific CaN-KO mice. Where indicated, MG132 was added $3 \mathrm{~h}$ before lysis. Data are expressed as mean $\pm \mathrm{SD}, 6$ independent cultures were used for both Ctr and Ctr treated with FK506 for 7 days, 5 for CaN-KO, and 3 for Ctr treated with FK506 for 1 day. ${ }^{*} p<0.01 ;{ }^{* *} p<0.01 ;{ }^{* *} p<0.001$, unpaired two-tailed Student's t-test.

\subsection{Upregulation of GLAST Protein in Astroglial CaN-KO Hippocampal Synaptosomes}

To consolidate our findings and to investigate if in vivo deletion of CaN may result in GLAST upregulation, we used WB to quantify GLAST protein in hippocampal synaptosomal fractions in which GLAST is enriched in perisynaptic astrocytic processes at tripartite synapse [20,21]. According to our in vitro data, GLAST was significantly up-regulated in synaptosomes from astroglial CaN-KO mouse compare with control preparations (Figure 5A), while no changes of GLAST mRNA levels were detected (Figure 5B). 


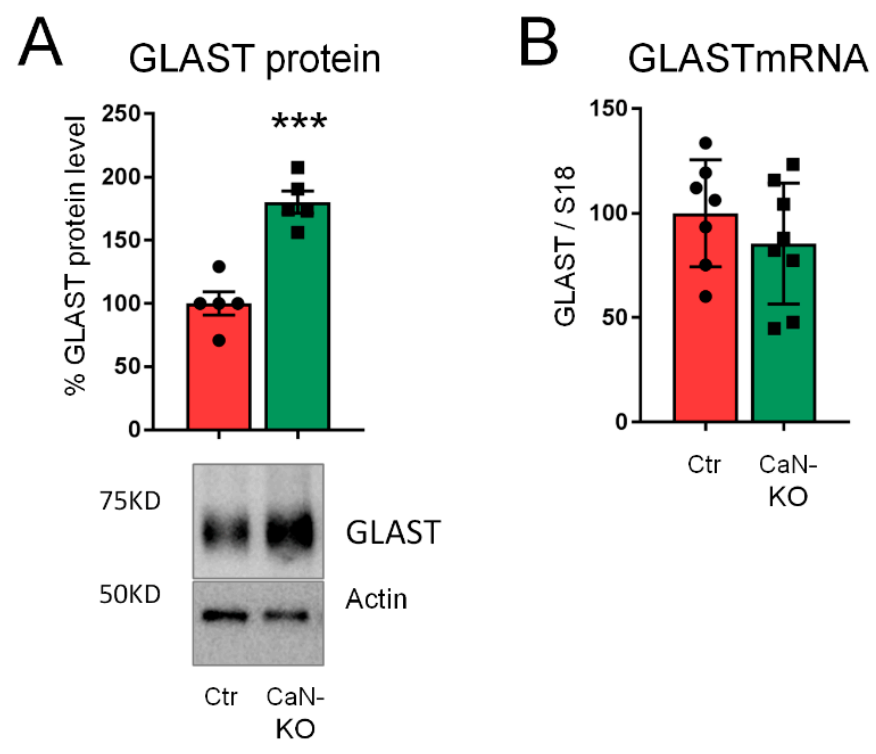

Figure 5. GLAST protein, but not mRNA, is upregulated in hippocampal synaptosomes from astroglial CaN-KO mouse. (A) GLAST protein expression in hippocampal synaptosomes from control (Ctr) and astroglial CaN-KO mice (CaN-KO). Data are expressed as mean $\pm \mathrm{SD}, 5$ independent synaptosomal preparations, each of which was a pool from two animals, were used for both Ctr and CaN-KO. *** $p<0.001$, unpaired two-tailed Student's t-test. (B) Real-time PCR of GLAST from hippocampi of $\mathrm{Ctr}$ and astrocytic CaN-KO mice. Values represent mean $\pm \mathrm{SD} \Delta \mathrm{C}(\mathrm{t})$ of gene/S18 of 7 independent samples for each genotype.

Altogether, our data suggest that inhibition of $\mathrm{CaN}$ in astrocytes results in deregulation of both protein synthesis and degradation, albeit with different time-scale, resulting in dynamic modulation of GLAST protein expression.

\section{Discussion}

The present work was designed to investigate the mechanism(s) of upregulation of glutamate/aspartate transporter GLAST in cultured hippocampal astrocytes [6]. We found that, while astrocyte-specific genetic ablation of $\mathrm{CaN}$ leads to a constitutive increase of GLAST protein, its pharmacological inhibition produces a biphasic modulation showing reduction at 1-2 days but upregulation of GLAST protein expression at 7th day after beginning of treatment. Such a modulation occurred at a posttranscriptional level through differential inhibition of protein synthesis and degradation (Figure 6).

Uptake of glutamate, released during neuronal activity and synaptic transmission, is an important aspect of astroglial patho-physiology [3] and the fine tuning of this process by regulation of expression of glutamate transporters contributes to glutamate homeostasis, while deregulation of glutamate transporters expression in many pathological conditions has been documented [9,10]. Expression of GLAST has been shown to be modulated by several physiologically and pathologically relevant agents. Thus, growth factors and steroid hormones upregulate GLAST in cultured astrocytes, at both mRNA and protein levels [22,23]. Instead, phorbol esthers and thrombin downregulate GLAST in cultured astrocytes [24-26]. In neuron-astrocyte co-cultures, GLAST expression has been shown to be dynamically modulated by neuronal activity and depended on the state of neuronal maturation [27]. Such a modulation has been proposed to depend on astrocyte's interaction with substrate and other cells [28], while whether this requires de-novo GLAST mRNA synthesis is not known. However, it has been demonstrated that transcriptional upregulation of GLAST in response to amitriptyline requires activation of transcription factor NF-kB [29] and that GLAST promoter contains NF-kB binding sites [30]. We and others have shown that, in astrocytes, cytokine- and $A \beta$-induced activation of 
NF-kB requires activation of CaN [31-33], although, whether CaN activation of NF-kB is required for regulation of GLAST expression is not clear. Here we show that both in vitro and in vivo blockade of CaN activity by pharmacological and genetic means leads to modulation of GLAST expression without changes of its mRNA levels, suggesting a posttranscriptional mechanism. However, CaN inhibition may not necessarily affect same pathways induced by CaN over-activation. Thus, pathology-related over-activation of $\mathrm{CaN}$ in astrocytes has been repeatedly associated with downstream transcriptional remodeling [12,31,34]. Therefore, it could be speculated that transcriptional regulation of GLAST may be activated in conditions of experimental and/or pathological over-activation of $\mathrm{CaN}$ in astrocytes, while in resting/physiological conditions the regulation occurs through CaN modulation of protein synthesis and degradation, although experimental proof of this speculation is a matter of future work.

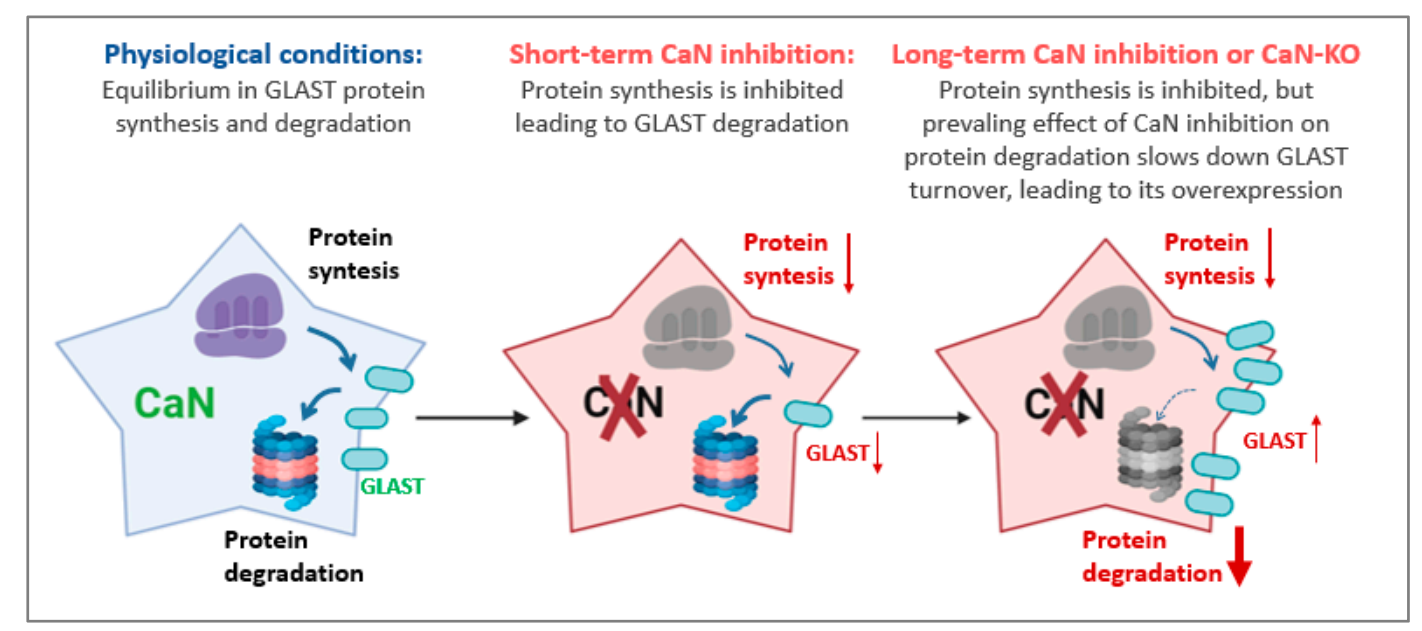

Figure 6. Scheme of the proposed mechanism for the time-dependent dynamic modulation of GLAST protein expression upon inhibition of $\mathrm{CaN}$ in astrocytes. Left astrocyte, protein synthesis and protein degradation are in equilibrium maintaining constant expression of GLAST. Middle astrocyte, short-term CaN inhibition by FK506 results in a prevalent inhibition of protein synthesis resulting in downregulation of GLAST. Right astrocyte, long-term pharmacological CaN inhibition or knock-out of CaN in astrocytes result in prevailing effect on the inhibition of protein degradation over the inhibition of protein synthesis, resulting in slowing down the GLAST turnover and its accumulation. The time-dependent effect of CaN inhibition on dynamics of other proteins in the cell may differ from that of GLAST.

The central finding of this work is that both genetic ablation and pharmacological inhibition of $\mathrm{CaN}$ drastically inhibited both protein synthesis and degradation in cultured astrocytes. Here we investigate this focusing on GLAST expression. However, there may be more general interpretation of this finding. The expression dynamics of other than GLAST proteins may not necessarily be modulated as those of GLAST. This may depend on the posttranslational modifications and on the protein turnover rate [35]. Therefore, it may be suggested that the inhibition of CaN astrocytes will likely result in dysproteostasis [17] rather than massive decrease/increase in protein mass, i.e., some proteins will be upregulated while some downregulated albeit with different time-scale dynamics, yet other proteins will not change their expression levels. This may be reflected by the fact that at a given total proteins concentration in our samples, actin expression did not change at any condition and time-point.

Taken separately, involvement of $\mathrm{CaN}$ in protein synthesis and degradation processes is not novel. Thus, direct $\mathrm{CaN}$ modulation of protein synthesis has been reported previously and, at the level of translational machinery, at least four factors, eukaryotic translation initiation factor 2B (eIF2B), eukaryotic translation initiation factor $4 \mathrm{E}$ binding protein 1 (4EBP1), eukaryotic translation initiation factor $4 \mathrm{~F}(\mathrm{eIF} 4 \mathrm{~F})$ and eukaryotic translation elongation factor 2 (eEF2), have been suggested to be directly regulated by $\mathrm{CaN}$ [36]. Protein degradation has also been associated with $\mathrm{CaN}$ activity through its involvement in lysosomal activity [37], autophagy [38,39], ER stress, and unfolded protein response 
(UPR) [40,41]. Association of CaN activation which ubiquitin-ligase/proteasome system has also been suggested [42,43]. In astrocytes, $\mathrm{CaN}$ has been shown to regulate UPR through direct interaction with protein kinase RNA-like endoplasmic reticulum kinase (PERK) [44,45]. Recently, activation of PERK-mediated UPR in astrocytes has been shown to induce neuronal degeneration [46]. As it was mentioned above, astrocytes are the main homeostatic cells in the CNS, which use the cell-cell interaction-mediated $\mathrm{Ca}^{2+}$ signaling to finely tune their homeostatic activities [47]. In light of this, and of the discussed above pathways which may be affected by the over-activation of $\mathrm{CaN}$, it can be speculated that, in neurodegenerative conditions, the astroglial $\mathrm{CaN}$ over-activation may result in a pattern of protein expression, at least in part, similar to that produced by the inhibition of CaN in astrocytes, albeit, this may differ substantially between brain cell types. Such an effect may be brought by the concomitance of the $\mathrm{CaN}$ activation with other known and unknown mechanisms, affecting the proteostasis machinery, which are activated by the neurodegenerative process. One could suggest that the neuropathological outcome of the disease will not depend on the action of a single molecule/enzyme (e.g., over-activated $\mathrm{CaN}$ ), but on the result of such an intricate interaction with parallel/downstream mechanisms bringing the proteostasis machinery to collapse.

In conclusion, our results suggest that astroglial $\mathrm{CaN}$, through modulation of a dynamic equilibrium between protein synthesis and degradation, may regulate proteostasis of astrocytes and thereby, their homeostatic activities including uptake of glutamate, in which GLAST is primarily involved. This provides a framework for investigation of the role of astroglial $\mathrm{CaN}$ in regulation of the CNS proteostasis.

\section{Materials and Methods}

\subsection{Astrocyte-Specific CaN KO Mice}

Generation and handling of conditional CaN knockout (KO) in GFAP-expressing astrocytes has been described previously [6]. Mice were housed in the animal facility of the Università del Piemonte Orientale, with unlimited access to water and food. Animals were managed in accordance with European directive 2010/63/UE and with Italian law D.1. 26/2014. The procedures were approved (3 June 2016) by the local animal-health and ethical committee (Università del Piemonte Orientale) and were authorized by the national authority (Istituto Superiore di Sanità; authorization numbers N. 77-2017 and N. 214-2019). All efforts were made to reduce the number of animals by following the $3 R$ 's rule.

\subsection{Mouse Astrocytic Primary Cultures}

Primary astroglial cultures were obtained by extracting hippocampi from either ACN-Ctr or KO mouse pups at postnatal day 1-5 (P1-P5). Hippocampi were dissected, in cold HBSS, from pups brains and dissociated by incubation with trypsin $\left(0.5 \mathrm{mg} / \mathrm{mL}, 37^{\circ} \mathrm{C}, 20 \mathrm{~min}\right)$ followed be a gentle triturating and resuspension in Dulbecco's Modified Eagle's Medium (DMEM, Sigma, Milan, Italy) — high glucose, supplemented with $10 \%$ foetal bovine serum (FBS), $2 \mathrm{mg} / \mathrm{mL}$ glutamine, $10 \mathrm{U} / \mathrm{mL}$ penicillin and $100 \mathrm{mg} / \mathrm{mL}$ streptomycin (Sigma, Milan, Italy). Each pup was processed separately and plated pre-treated with $0.1 \mathrm{mg} / \mathrm{mL}$ poly-L-lysine (PLL, Sigma, Milan, Italy). At sub-confluence (5-10 days in vitro), cells were detached with trypsin and pleated for experiments.

\subsection{Fetal Human Primary Astrocytes}

Primary fetal astrocyte-enriched cell cultures were derived from human fetal brain tissue (14-20 weeks of gestation) obtained from medically induced abortions. All material was collected from donors from whom a written informed consent for the use of the material for research purposes. Tissue was obtained in accordance with the Declaration of Helsinki and the Amsterdam UMC Research Code provided by the Medical Ethics Committee. Tissue samples were collected in astrocyte medium: DMEM/HAM F10 (1:1; Gibco/ThermoFisher Scientific, Waltham, MA, USA), supplemented with 
100 units/mL penicillin, $100 \mu \mathrm{g} / \mathrm{mL}$ streptomycin and $10 \%$ fetal calf serum (Gibco, Life Technologies, Grand Island, NY, USA). Cell isolation was performed as previously described [48].

\subsection{Pharmacological Treatments}

Cultured astrocytes were plated in a 6 well plate pre-treated with $0.1 \mathrm{mg} / \mathrm{mL}$ poly-L-lysine (PLL, Sigma, Milan, Italy), and grown until 60\% of confluence. 200 nM FK506 (TOCRIS, Cat. 3631) was added $24 \mathrm{~h}, 48 \mathrm{~h}$ or 1 week prior to lysis. In the 1 week treatment setting FK506 was re-added at 3rd day of treatment.

For assessment of proteasome activity, $3 \mathrm{~h}$ before lysis, cells were treated with $1 \mu \mathrm{M}(\mathrm{R})-\mathrm{MG} 132$ (Cat. 6033, TOCRIS, Britol, UK).

\subsection{Puromycin Incorporation Method (Surface Sensing of Translation, SUnSET)}

Cultured astrocytes were incubated with $4 \mu \mathrm{M}$ puromycin dihydrochloride (Cat. P8833, Sigma, Milan, Italy) supplemented in normal medium at $37^{\circ} \mathrm{C}$ with $5 \% \mathrm{CO}_{2}$ for $3 \mathrm{~h}$ [16]. Subsequently, cell's lysates were subjected to Western blot assay or fixed for immuno-histochemistry.

\subsection{Immunofluorescence and Confocal Microscopy}

Primary mouse hippocampal and foetal human astrocytes, grown on $13 \mathrm{~mm}$ glass coverslips and treated as indicated, were fixed in $4 \%$ formaldehyde and $4 \%$ sucrose, permeabilized $(7 \mathrm{~min}$ in $0.1 \%$ Triton X-100 in phosphate-buffered saline (PBS)), blocked in 1\% gelatin, and immunoprobed with an appropriate primary antibody (diluted in PBS supplemented with $1 \%$ gelatine) over night at $4{ }^{\circ} \mathrm{C}$. After 3 times washing in PBS, an Alexa-conjugated secondary antibody (1:300 in PBS supplemented with $1 \%$ gelatine) was applied for $1 \mathrm{~h}$ at room temperature (RT). The following primary antibodies were used: anti-GLAST (rabbit, 1:100, Cat. NB100-1869, Novusbio, Abingdon, UK), anti-puromycin (1:200, Millipore, Cat. MABE343). Secondary antibodies were as follows: Alexa Fluor 488 anti-mouse IgG, Alexa Fluor 555 anti-rabbit IgG (all secondary antibodies were from Molecular Probes, Life Technologies, Monza, Italy). Nuclei were counter-stained with 4',6-diamidino-2-phenylindole (DAPI). Images were acquired using an FV-500 Olympus (Tokyo, Japan) laser confocal scanning system with a 60X oil immersion objective. Immunofluorescence signal intensity per cell was measured with NIH ImageJ software v1.52p and was calculated as corrected total cell fluorescence (CTCF) $=$ Integrated Density-(Area of selected cell X Mean fluorescence of background readings).

\subsection{Preparation of Synaptosomes}

Synaptosomal fractions were isolated by differential centrifugation using standard protocols [49]. Briefly, mice were anesthetized and sacrificed by decapitation. Hippocampi were rapidly dissected and placed into ice-cold homogenization buffer containing $50 \mathrm{mM}$ MOPS, pH 7.4, $320 \mathrm{mM}$ sucrose, $0.2 \mathrm{mM}$ DTT, $100 \mathrm{mM} \mathrm{KCl}, 0.5 \mathrm{mM} \mathrm{MgCl} 2,0.01 \mathrm{mM}$ EDTA, and $1 \mathrm{mM}$ EGTA, protease inhibitor cocktails (PIC, Calbiochem, San Diego, CA, USA) and phosphatase inhibitor $\mathrm{Na}_{3} \mathrm{VO}_{4} 1 \mu \mathrm{M}$. All subsequent steps were performed at $4{ }^{\circ} \mathrm{C}$. The hippocampus were microdissected and homogenized in 1:10 w/v homogenization buffer with 12 strokes in a Teflon-glass douncer. The homogenates centrifuged for $10 \mathrm{~min}$ at $800 \times \mathrm{g}$ followed by centrifugation of the supernatant at $9200 \times \mathrm{g}$ for $15 \mathrm{~min}$. The resulting P2 pellet, representing the crude synaptosomal fraction, was solubilized in lysis buffer.

\subsection{Cell lysis and Western blot}

Astroglial cultures were lysed with $100 \mu \mathrm{L}$ of lysis buffer (50 mM TrisHCl (pH 67.4), sodium dodecyl sulphate (SDS) $0.5 \%, 5 \mathrm{mM}$ EDTA), $10 \mu \mathrm{L}$ of protease inhibitors cocktail (PIC, Calbiochem) and $1 \mu \mathrm{L}$ of phosphatase inhibitor Na3VO4 $1 \mathrm{M}$; removed with a scraper, and collected in a $1.5 \mathrm{~mL}$ tube. Lysates were then boiled for 5', and quantified with QuantiPro BCA Assay Kit (cat. SLBF3463, Sigma, Milan, Italy). 
$30 \mu \mathrm{g}$ of proteins were mixed with the right amount of Laemmli Sample Buffer 4X (Bio-Rad, Segrate, Italy), and boiled for $5^{\prime}$. Then samples were loaded on a $12 \%$ polyacrylamide-sodium dodecyl sulphate gel for electrophoresis. Proteins were transferred onto nitrocellulose membrane, using Mini Transfer Packs or Midi Transfer Packs, with Trans-Blot@Turbo TM (Bio-Rad, Segrate, Italy) according to manufacturer's instructions (Bio-Rad, Segrate, Italy).

The membrane was blocked in 5\% skim milk (Cat. 70166, Sigma, Milan, Italy) for 45' at room temperature. Subsequently membrane was incubated with indicated primary antibody, overnight at $4{ }^{\circ} \mathrm{C}$ in agitation. Primary antibodies used were: anti-GLAST (rabbit, 1:500, Cat. NB100-1869, Novusbio, Abingdon, UK), anti-puromycin (1:1000, Cat. MABE343, Millipore, Darmstadt, Germany), anti- $\beta$-Actin (mouse, 1:800, Sigma, Cat. A1978, Milan, Italy) was used to normalize protein load. Goat anti-mouse IgG $(\mathrm{H}+\mathrm{L})$ horseradisch peroxidase-conjugated secondary antibody (1:5000; Cat. 170-6516, Bio-Rad, Segrate, Italy) and Goat anti-mouse Igg $(\mathrm{H}+\mathrm{L})$ horseradish peroxidase-conjugated secondary antibody (1:5000; Cat. 170-6515, Bio-Rad, Segrate, Italy). Detection was carried out with SuperSignalTM West Pico PLUS Chemiluminescent Sbustrate (Thermo Scientific, Milan, Italy), based on the chemiluminescence of luminol and developed using ChemiDocTM Imaging System (Bio-Rad, Segrate, Italy). Quantitative densitometry of protein bands analysis was performed with ImageLab software.

\subsection{RNA Extraction and Real-Time PCR}

Total mRNA was extracted from $1.0 \times 10^{6}$ cells using TRIzol Lysis Reagent (Cat. 15596026, Invitrogen, Milan, Italy) according to manufacturer's instruction. First strand of cDNA was synthesized from $0.5-1 \mu \mathrm{g}$ of total RNA using SensiFast kit (BioLine, London, UK, Cat. BIO-65054). Real-Time PCR was performed using iTaq qPCR master mix according to manufacturer's instructions (Cat. 1725124, Bio-Rad, Segrate, Italy) on a SFX96 Real-time system (Bio-Rad, Segrate Italy). To normalize raw real time PCR data, S18 ribosomal subunit was used. Primer sequences are provided in Table 1. Data are expressed as delta-C(t) of gene of interest to S18 allowing appreciation of single gene expression level.

Table 1. List of oligonucleotide primers used for real-time PCR.

\begin{tabular}{cccc}
\hline Protein Name & Gene Name & \multicolumn{1}{c}{ Forward/Reverse } & Accession No. \\
\hline S18 & \multirow{2}{*}{ Rps18 } & TGCGAGTACTCAACACCAACA & NM_011296 \\
mouse and human & & CTGCTTTCCTCAACACCACA & NM_022551.3 \\
EAAT1/GLAST mouse & Slc1a3 & AATGCCTTCGTTCTGCTCAC & NM_148938 \\
& \multirow{2}{*}{ Slc1a3 } & GTTGCTCATGAGAAGCTCCCC & GGAATCACCCACAGAAAGCC \\
& & NM_004172.4 \\
\hline
\end{tabular}

\subsection{Statistical Analysis}

Statistical analysis and related graphical representations was done using GraphPad Prism v.7. A two-tailed unpaired Student's t-test or one way ANOVA test ware used. Differences were considered significant at $p<0.05$.

Author Contributions: Conceptualization, G.D., D.L. and L.T.; Methodology, G.D, D.L., E.A. and L.T.; Investigation, G.D., E.R. and L.T.; Data Curation, G.D., E.R. and L.T.; Writing-Original Draft Preparation, G.D, L.T., and D.L.; Writing-Review and Editing, G.D, E.A., A.A.G., L.T., and D.L.; Supervision, R.C., E.A. and A.A.G.; Funding Acquisition, A.A.G. and D.L. All authors have read and agreed to the published version of the manuscript.

Funding: This work had the following financial supports: grants 2013-0795 to AAG, 2014-1094 to DL from the Fondazione Cariplo; grants FAR-2016 and FAR-2019 to DL from The Università del Piemonte Orientale; L.T. was supported by fellowship from the CRT Foundation (1393-2017).

Acknowledgments: We acknowledge the HIS Mouse Facility of the Academic Medical Center, Amsterdam and the Bloemenhove Clinic (Heemstede, The Netherlands) for providing fetal tissues and Jasper Anink (Department of Neuropathology, Amsterdam) for his technical support.

Conflicts of Interest: The authors declare no conflict of interest. 


\section{References}

1. Danbolt, N.C. Glutamate uptake. Prog. Neurobiol. 2001, 65, 1-105. [CrossRef]

2. Nicholls, D.; Attwell, D. The release and uptake of excitatory amino acids. Trends Pharmacol. Sci. 1990, 11, 462-468. [CrossRef]

3. Malik, A.R.; Willnow, T.E. Excitatory Amino Acid Transporters in Physiology and Disorders of the Central Nervous System. Int. J. Mol. Sci. 2019, 20, 5671. [CrossRef]

4. Garlin, A.B.; Sinor, A.D.; Sinor, J.D.; Jee, S.H.; Grinspan, J.B.; Robinson, M.B. Pharmacology of sodium-dependent high-affinity L-[3H]glutamate transport in glial cultures. J. Neurochem. 1995, 64, 2572-2580. [CrossRef]

5. Swanson, R.A.; Liu, J.; Miller, J.W.; Rothstein, J.D.; Farrell, K.; Stein, B.A.; Longuemare, M.C. Neuronal regulation of glutamate transporter subtype expression in astrocytes. J. Neurosci. Off. J. Soc. Neurosci. 1997, 17, 932-940. [CrossRef]

6. Tapella, L.; Soda, T.; Mapelli, L.; Bortolotto, V.; Bondi, H.; Ruffinatti, F.A.; Dematteis, G.; Stevano, A.; Dionisi, M.; Ummarino, S.; et al. Deletion of calcineurin from GFAP-expressing astrocytes impairs excitability of cerebellar and hippocampal neurons through astroglial Na+/K+ ATPase. Glia 2020, 68, 543-560. [CrossRef]

7. Pajarillo, E.; Rizor, A.; Lee, J.; Aschner, M.; Lee, E. The role of astrocytic glutamate transporters GLT-1 and GLAST in neurological disorders: Potential targets for neurotherapeutics. Neuropharmacology 2019, 161, 107559. [CrossRef]

8. Bristot Silvestrin, R.; Bambini-Junior, V.; Galland, F.; Daniele Bobermim, L.; Quincozes-Santos, A.; Torres Abib, R.; Zanotto, C.; Batassini, C.; Brolese, G.; Gonçalves, C.-A.; et al. Animal model of autism induced by prenatal exposure to valproate: altered glutamate metabolism in the hippocampus. Brain Res. 2013, 1495, 52-60. [CrossRef]

9. Martinez-Lozada, Z.; Guillem, A.M.; Robinson, M.B. Transcriptional Regulation of Glutamate Transporters: From Extracellular Signals to Transcription Factors. Adv. Pharmacol. San Diego Calif 2016, 76, 103-145.

10. Lee, E.; Karki, P.; Johnson, J.; Hong, P.; Aschner, M. Manganese Control of Glutamate Transporters' Gene Expression. Adv. Neurobiol. 2017, 16, 1-12.

11. Abdul, H.M.; Sama, M.A.; Furman, J.L.; Mathis, D.M.; Beckett, T.L.; Weidner, A.M.; Patel, E.S.; Baig, I.; Murphy, M.P.; LeVine, H.; et al. Cognitive decline in Alzheimer's disease is associated with selective changes in calcineurin/NFAT signaling. J. Neurosci. Off. J. Soc. Neurosci. 2009, 29, 12957-12969. [CrossRef]

12. Lim, D.; Rocchio, F.; Lisa, M.; Fcancesco, M. From Pathology to Physiology of Calcineurin Signalling in Astrocytes. Opera Med. Physiol. 2016, 2, 43-61.

13. Rao, A.; Luo, C.; Hogan, P.G. Transcription factors of the NFAT family: regulation and function. Annu. Rev. Immunol. 1997, 15, 707-747. [CrossRef]

14. Lee, J.I.; Burckart, G.J. Nuclear factor kappa B: important transcription factor and therapeutic target. J. Clin. Pharmacol. 1998, 38, 981-993. [CrossRef]

15. Li, H.; Rao, A.; Hogan, P.G. Interaction of calcineurin with substrates and targeting proteins. Trends Cell Biol. 2011, 21, 91-103. [CrossRef]

16. Schmidt, E.K.; Clavarino, G.; Ceppi, M.; Pierre, P. SUnSET, a nonradioactive method to monitor protein synthesis. Nat. Methods 2009, 6, 275-277. [CrossRef]

17. Hipp, M.S.; Kasturi, P.; Hartl, F.U. The proteostasis network and its decline in ageing. Nat. Rev. Mol. Cell Biol. 2019, 20, 421-435. [CrossRef]

18. Rousseau, A.; Bertolotti, A. Regulation of proteasome assembly and activity in health and disease. Nat. Rev. Mol. Cell Biol. 2018, 19, 697-712. [CrossRef]

19. Lee, D.H.; Goldberg, A.L. Proteasome inhibitors: valuable new tools for cell biologists. Trends Cell Biol. 1998, 8, 397-403. [CrossRef]

20. Chaudhry, F.A.; Lehre, K.P.; van Lookeren Campagne, M.; Ottersen, O.P.; Danbolt, N.C.; Storm-Mathisen, J. Glutamate transporters in glial plasma membranes: highly differentiated localizations revealed by quantitative ultrastructural immunocytochemistry. Neuron 1995, 15, 711-720. [CrossRef]

21. Rudy, C.C.; Hunsberger, H.C.; Weitzner, D.S.; Reed, M.N. The Role of the Tripartite Glutamatergic Synapse in the Pathophysiology of Alzheimer's Disease. Aging Dis. 2015, 6, 131-148. [CrossRef] [PubMed]

22. Pawlak, J.; Brito, V.; Küppers, E.; Beyer, C. Regulation of glutamate transporter GLAST and GLT-1 expression in astrocytes by estrogen. Brain Res. Mol. Brain Res. 2005, 138, 1-7. [CrossRef] [PubMed] 
23. Suzuki, K.; Ikegaya, Y.; Matsuura, S.; Kanai, Y.; Endou, H.; Matsuki, N. Transient upregulation of the glial glutamate transporter GLAST in response to fibroblast growth factor, insulin-like growth factor and epidermal growth factor in cultured astrocytes. J. Cell Sci. 2001, 114, 3717-3725. [PubMed]

24. Espinoza-Rojo, M.; López-Bayghen, E.; Ortega, A. GLAST: gene expression regulation by phorbol esters. Neuroreport 2000, 11, 2827-2832. [CrossRef]

25. Wang, Z.; Li, W.; Mitchell, C.K.; Carter-Dawson, L. Activation of protein kinase C reduces GLAST in the plasma membrane of rat Müller cells in primary culture. Vis. Neurosci. 2003, 20, 611-619. [CrossRef]

26. Piao, C.; Ralay Ranaivo, H.; Rusie, A.; Wadhwani, N.; Koh, S.; Wainwright, M.S. Thrombin decreases expression of the glutamate transporter GLAST and inhibits glutamate uptake in primary cortical astrocytes via the Rho kinase pathway. Exp. Neurol. 2015, 273, 288-300. [CrossRef]

27. Perego, C.; Vanoni, C.; Bossi, M.; Massari, S.; Basudev, H.; Longhi, R.; Pietrini, G. The GLT-1 and GLAST glutamate transporters are expressed on morphologically distinct astrocytes and regulated by neuronal activity in primary hippocampal cocultures. J. Neurochem. 2000, 75, 1076-1084. [CrossRef]

28. Cho, S.; Muthukumar, A.K.; Stork, T.; Coutinho-Budd, J.C.; Freeman, M.R. Focal adhesion molecules regulate astrocyte morphology and glutamate transporters to suppress seizure-like behavior. Proc. Natl. Acad. Sci. USA 2018, 115, 11316-11321. [CrossRef]

29. Tai, Y.-H.; Tsai, R.-Y.; Wang, Y.-H.; Cherng, C.-H.; Tao, P.-L.; Liu, T.-M.; Wong, C.-S. Amitriptyline induces nuclear transcription factor-kappaB-dependent glutamate transporter upregulation in chronic morphine-infused rats. Neuroscience 2008, 153, 823-831. [CrossRef]

30. Karki, P.; Kim, C.; Smith, K.; Son, D.-S.; Aschner, M.; Lee, E. Transcriptional Regulation of the Astrocytic Excitatory Amino Acid Transporter 1 (EAAT1) via NF- $\mathrm{kB}$ and Yin Yang 1 (YY1). J. Biol. Chem. 2015, 290, 23725-23737. [CrossRef]

31. Fernandez, A.M.; Fernandez, S.; Carrero, P.; Garcia-Garcia, M.; Torres-Aleman, I. Calcineurin in reactive astrocytes plays a key role in the interplay between proinflammatory and anti-inflammatory signals. J. Neurosci. Off. J. Soc. Neurosci. 2007, 27, 8745-8756. [CrossRef] [PubMed]

32. Fernandez, A.M.; Jimenez, S.; Mecha, M.; Dávila, D.; Guaza, C.; Vitorica, J.; Torres-Aleman, I. Regulation of the phosphatase calcineurin by insulin-like growth factor I unveils a key role of astrocytes in Alzheimer's pathology. Mol. Psychiatry 2012, 17, 705-718. [CrossRef] [PubMed]

33. Lim, D.; Iyer, A.; Ronco, V.; Grolla, A.A.; Canonico, P.L.; Aronica, E.; Genazzani, A.A. Amyloid beta deregulates astroglial mGluR5-mediated calcium signaling via calcineurin and Nf-kB. Glia 2013, 61, 1134-1145. [CrossRef] [PubMed]

34. Furman, J.L.; Norris, C.M. Calcineurin and glial signaling: neuroinflammation and beyond. J. Neuroinflammation 2014, 11, 158. [CrossRef] [PubMed]

35. Basisty, N.; Meyer, J.G.; Schilling, B. Protein Turnover in Aging and Longevity. Proteomics 2018, 18, e1700108. [CrossRef]

36. Sans, M.D.; Williams, J.A. Calcineurin is required for translational control of protein synthesis in rat pancreatic acini. Am. J. Physiol. Cell Physiol. 2004, 287, C310-C319. [CrossRef] [PubMed]

37. Aufschnaiter, A.; Kohler, V.; Büttner, S. Taking out the garbage: cathepsin D and calcineurin in neurodegeneration. Neural Regen. Res. 2017, 12, 1776-1779.

38. Medina, D.L.; Di Paola, S.; Peluso, I.; Armani, A.; De Stefani, D.; Venditti, R.; Montefusco, S.; Scotto-Rosato, A.; Prezioso, C.; Forrester, A.; et al. Lysosomal calcium signalling regulates autophagy through calcineurin and TFEB. Nat. Cell Biol. 2015, 17, 288-299. [CrossRef]

39. Lapierre, L.R.; Kumsta, C.; Sandri, M.; Ballabio, A.; Hansen, M. Transcriptional and epigenetic regulation of autophagy in aging. Autophagy 2015, 11,867-880. [CrossRef]

40. Mukherjee, A.; Soto, C. Role of calcineurin in neurodegeneration produced by misfolded proteins and endoplasmic reticulum stress. Curr. Opin. Cell Biol. 2011, 23, 223-230. [CrossRef]

41. Shah, S.Z.A.; Hussain, T.; Zhao, D.; Yang, L. A central role for calcineurin in protein misfolding neurodegenerative diseases. Cell. Mol. Life Sci. CMLS 2017, 74, 1061-1074. [CrossRef] [PubMed]

42. Li, H.-H.; Kedar, V.; Zhang, C.; McDonough, H.; Arya, R.; Wang, D.-Z.; Patterson, C. Atrogin-1/muscle atrophy F-box inhibits calcineurin-dependent cardiac hypertrophy by participating in an SCF ubiquitin ligase complex. J. Clin. Invest. 2004, 114, 1058-1071. [CrossRef] [PubMed] 
43. Shimizu, H.; Langenbacher, A.D.; Huang, J.; Wang, K.; Otto, G.; Geisler, R.; Wang, Y.; Chen, J.-N. The Calcineurin-FoxO-MuRF1 signaling pathway regulates myofibril integrity in cardiomyocytes. eLife 2017, 6, e27955. [CrossRef] [PubMed]

44. Bollo, M.; Paredes, R.M.; Holstein, D.; Zheleznova, N.; Camacho, P.; Lechleiter, J.D. Calcineurin interacts with PERK and dephosphorylates calnexin to relieve ER stress in mammals and frogs. PloS One 2010, 5, e11925. [CrossRef] [PubMed]

45. Chen, Y.; Holstein, D.M.; Aime, S.; Bollo, M.; Lechleiter, J.D. Calcineurin $\beta$ protects brain after injury by activating the unfolded protein response. Neurobiol. Dis. 2016, 94, 139-156. [CrossRef]

46. Smith, H.L.; Freeman, O.J.; Butcher, A.J.; Holmqvist, S.; Humoud, I.; Schätzl, T.; Hughes, D.T.; Verity, N.C.; Swinden, D.P.; Hayes, J.; et al. Astrocyte Unfolded Protein Response Induces a Specific Reactivity State that Causes Non-Cell-Autonomous Neuronal Degeneration. Neuron 2020, 105, 855-866. [CrossRef]

47. Khakh, B.S.; Sofroniew, M.V. Diversity of astrocyte functions and phenotypes in neural circuits. Nat. Neurosci. 2015, 18, 942-952. [CrossRef]

48. Korotkov, A.; Broekaart, D.W.M.; Banchaewa, L.; Pustjens, B.; van Scheppingen, J.; Anink, J.J.; Baayen, J.C.; Idema, S.; Gorter, J.A.; van Vliet, E.A.; et al. microRNA-132 is overexpressed in glia in temporal lobe epilepsy and reduces the expression of pro-epileptogenic factors in human cultured astrocytes. Glia 2020, 68, 60-75. [CrossRef]

49. Gillardon, F. Differential mitochondrial protein expression profiling in neurodegenerative diseases. Electrophoresis 2006, 27, 2814-2818. [CrossRef]

(C) 2020 by the authors. Licensee MDPI, Basel, Switzerland. This article is an open access article distributed under the terms and conditions of the Creative Commons Attribution (CC BY) license (http://creativecommons.org/licenses/by/4.0/). 